\title{
Force production capacity and functional reflex activity in young and elderly men*
}

\author{
Urs Granacher ${ }^{1,2}$, Markus Gruber ${ }^{3}$ and Albert Gollhofer ${ }^{4}$ \\ ${ }^{1}$ Institute of Exercise and Health Sciences, University of Basel, Switzerland, ${ }^{2}$ Institute of Sport Science, \\ Friedrich-Schiller-University Jena, Germany, ${ }^{3}$ Department of Training and Movement Science, University \\ of Potsdam, Germany, ${ }^{4}$ Institute of Sport and Sport Science, University of Freiburg, Germany \\ (Institute where the work was carried out)
}

ABSTRACT. Background and aims: There is growing evidence that biological aging results in impaired force production of the lower extremities and deficits in reflex activity. This study therefore investigated maximal and explosive force production capacity, functional reflex activity (FRA) during gait perturbations, and the relationship between variables of force production capacity and FRA in young and elderly men. Methods: Twenty-eight young (age $27 \pm 3$ yrs, $n=14$ ) and old (age $67 \pm 4$ yrs, $n=14$ ) healthy active men were tested for decelerating impulses on a treadmill and for their maximal isometric leg extension force (MIF) and rate of force development (RFD) on a leg-press. Results: MIF and RFD were significantly lower in the elderly than in younger participants (MIF $\sim 45 \%, \mathrm{p}<0.01 ; R F D \sim 50 \%, \mathrm{p}<0.01)$. Elderly subjects showed significant decreases in FRA in the prime mover which compensated for the decelerating impulse (FRA 29\%, p<0.05). No significant correlations were found between FRA, MIF or RFD. Conclusions: Lower MIF, $R F D$ and impaired FRA were found in old compared with young men. The absence of significant correlations between measures of strength performance and FRA may indicate that different mechanisms within the neuromuscular system are responsible for these capacities. This result may imply that force production and functional reflex activity are independent of each other and may have to be trained complementarily.

(Aging Clin Exp Res 2010; 22: 374-382)

${ }^{\circ}$ 2010, Editrice Kurtis

\section{INTRODUCTION}

There have been substantial increases in the percentage of people aged 65 and older in western industrial countries. This trend necessitates intense research into the effects of aging on neuromuscular performance and its functional consequences (e.g., increased fall incidence). Biologic aging results in unavoidable decreases in maximal and explosive force production capacity (1). Between the ages of 30 and 80 , the average reported decreases in maximal strength range from 20 to $40 \%$ (2), depending on study design (e.g., cross-sectional us longitudinal [3]), applied methods (isokinetic vs isometric [4]), tested muscle groups (upper us lower extremity [5]), investigated age groups (old vs oldest old [6]), health status (vigorous vs frail [7]), and fitness level (sedentary vs active [8]). Age-related decreases in explosive force production capacity exceed those in maximal strength, the most severe losses occurring between the seventh and ninth decade of life (6). McNeil et al. (6) observed a 25\% decrease in power of the dorsiflexors between the third and seventh decade of life, which doubled in the next two decades, so that men in their ninth decade of life produced $60 \%$ less power than young men (mean $26 \mathrm{yrs}$ ).

The impact of neuromuscular aging is not only restricted to strength and power performance, but also has an effect on static and dynamic postural control (9, 10). Era et al. (9) assessed static postural control on a force platform in a randomly selected sample of subjects aged 30 years and older. They observed that deterioration of postural control mechanisms start relatively early in life. Differences in balance performance were already apparent between young (30-39 yrs) and middleaged adults (40-49 yrs) and became even more pronounced after the age of 60 . In terms of dynamic postural control, Pavol et al. (10) investigated fall incidence in old vs young subjects when confronted with unexpected

\footnotetext{
*This manuscript was presented as a slide show at the 55th Annual Meeting of the ACSM, Indianapolis (USA), 2008 (Granacher U, Strass D, Gollhofer A. Effect of aging on power output and functional reflex activity. Med Sci Sports Exerc 2008; 40: 87-88).

Key words: Explosive force, gait perturbations, maximal force, postural control, seniors.

Correspondence: Urs Granacher, University of Basel, Institute of Exercise and Health Sciences, Birsstr. $320 \mathrm{~B}, 4052$ Basel, Switzerland.

E-mail: urs.granacher@unibas.ch

Received February 26, 2009; accepted in revised form November 10, 2009.

First published ahead of print November 27, 2009 as DOI: 10.3275/6706
} 
slips during a sit-to-stand task, and observed that older adults are more likely to fall at exposure to initial, unexpected perturbation.

From a functional point of view, it is of great interest to know whether there is a relationship between maximal and explosive strength performance and static and dynamic postural control in old age. In this regard, Izquierdo et al. (1) were able to show that there is an association between static and dynamic postural control measured by computerized dynamic posturography and maximal rate of force development of the leg extensors measured under isometric conditions. Pijnappels et al. (11) also identified maximum isometric push-off force in a leg-press as best measure to distinguish between elderly individuals who would fall after a gait perturbation and those who recovered their balance. In addition, Pavol et al. (12) found an association between decreased lower extremity strength and increased risk of falling after tripping. Thus, it may be argued that a causal relationship between lower extremity strength performance and the inability to recover after a postural perturbation has been established by kinetic analyses $(11,12)$. However, to the authors' knowledge, there is no study available which investigated a possible correlation between force production capacity and functional reflex activity (FRA $)^{1}$ during gait perturbations in old age by means of kinetic and electromyographic (EMG) analyses. Therefore, this study aimed at investigating in a cohort of young and elderly men 1) maximal and explosive force production, with special emphasis on the early part of the force-time curve; 2) FRA, examined by unexpected treadmill perturbations; 3 ) the relationship between measures of force production and FRA during gait perturbations.

\section{METHODS}

\section{Study population}

Twenty-eight young (age $27 \pm 3$ yrs, body mass $74 \pm 6$ $\mathrm{kg}$, body height $178 \pm 6 \mathrm{~cm}$, BMI $24 \pm 1 \mathrm{~kg} / \mathrm{m}^{2}, \mathrm{n}=14$ ) and elderly men (age $67 \pm 4$ yrs, body mass $77 \pm 5 \mathrm{~kg}$, body height $175 \pm 5 \mathrm{~cm}$, BMI $25 \pm 2 \mathrm{~kg} / \mathrm{m}^{2}, \mathrm{n}=14$ ) provided their written informed consent to participate in the study after the experimental procedures had been explained. Subjects were healthy men with no history of serious muscular, neurological, cardiovascular, metabolic or inflammatory diseases. Before the study tests started, participants were asked to complete the validated "Freiburg questionnaire for everyday and sports activities ${ }^{\complement}$ " (14). Young and elderly subjects were classified as physically active (young $13 \pm 8 \mathrm{~h} /$ week, old $13 \pm 3 \mathrm{~h} /$ week). Ethical permission was given by the University of Freiburg, Germany, and all experiments were conducted according to the Declaration of Helsinki.

\section{Testing protocol}

In order to analyse possible age-related impairments in FRA during gait perturbations and in maximal and explosive force production, all subjects were tested for 1 ) treadmill perturbation impulses and 2) maximal isometric leg extension force on a leg-press (MIF). On the basis of these results, correlations between strength performance and FRA were calculated. This testing sequence was applied in order to keep the effects of neuromuscular fatigue to the minimum.

\section{Treadmill perturbation}

Before the actual testing started, subjects were provided with standardized low-cut athletic footwear (Adidas ${ }^{\circledR}$, Spezial, Herzogenaurach, Germany) to minimize variability and to control for shoe type and design. Thereafter, participants were accustomed to walking on a treadmill for a 5-min warm-up period. Decelerating perturbation impulses were randomly induced at separate phases of the gait cycle while subjects were walking on a treadmill (Woodway ${ }^{\circledR}$, Weil am Rhein, Germany). In this way, an everyday stumbling situation was transferred into artificial laboratory conditions. For safety reasons, subjects wore a chest harness system. The treadmill was powered by a DC motor with a gear unit, tachometer generator, and transistor-servo-amplifier in a four-quadrant system. The impulse capacity of the amplifier (MTR-7025) produced $12.750 \mathrm{~W}$ for $1 \mathrm{~s}$. This allowed rapid decelerating impulses of the treadmill from $3.5 \mathrm{~km} / \mathrm{h}$ to a backward velocity of $0.6 \mathrm{~km} / \mathrm{h}$ within $0.4 \mathrm{~s}$. Therefore, the treadmill was not only decelerated to zero, but the direction of the belt movement was also reversed. Electrical switches implemented in inlays of the right shoe (underneath the heel and the ball of the foot) were used as triggers to decelerate the treadmill precisely at heel contact and to determine the stance and swing phase of a regular gait cycle. The decelerating perturbation impulses were time-analysed 120 ms after the first biomechanical response to the perturbation impulse, in order to determine perturbation-induced reflex responses. The first biomechanical response to the perturbation impulse (trigger) was indicated by a goniometer signal. An electronic two-axial goniometer (Penny \& Giles ${ }^{\circledR}$, type XM180, Gwent, UK) was therefore firmly attached with double-sided adhesive tape along the Achilles tendon at the ankle joint. The gonio signal was filtered by a Butterworth low-pass filter, at a cut-off frequency of $10 \mathrm{~Hz}$. With regard to the decelerating impulse, the inflection point of the gonio-signal for plantar/dorsi flexion of the ankle was calculated and taken as a trigger for further data analysis (Fig. 1). The maximal angular velocity of the ankle joint was determined in the

\footnotetext{
${ }^{1}$ FRA refers to reflexive (involuntary) activation of muscles of the lower extremities, for the purpose of compensating for gait perturbations while walking on a treadmill (13).
} 
time-interval $120 \mathrm{~ms}$ after the first biomechanical response to the perturbation impulse. Latency, defined as the time elapsing between the first biomechanical response in the gonio-signal and the first EMG response, was analysed. Altogether, a total of ten decelerating impulses on the treadmill were applied and averaged for further data analysis. Besides the perturbation situations, the normal walking pattern (during unperturbed walking) was recorded at a speed of $3.5 \mathrm{~km} / \mathrm{h}$. This protocol has recently been described in detail elsewhere $(13,15)$. Pijnappels et al. (16) also investigated EMG modulation in anticipation of possible tripping during walking in young and older adults, and reported that anticipatory effects are not expected to jeopardize the validity of experiments in which subjects are perturbed more than once.

\section{Leg-press}

MIF was measured on a leg-press, with each foot resting on a separate one-dimensional force platform (Kistler ${ }^{\circledR}$, Winterthur, Switzerland). Subjects were horizontally positioned on the sledge of the leg-press, with hip and knee angles adjusted at $90^{\circ}$. The waist was fixed and subjects were allowed to stabilize their upper body by holding on to handles attached to the leg-press. Subjects were instructed to avoid forced respiration during maximal efforts. Before testing started, subjects became accustomed to the testing procedure by a warm-up of 3-5 submaximal isometric actions. The intensity of submaximal contractions was controlled on the Borg Scale. Thereafter, each subject performed 3-4 leg-press exercises with maximal voluntary effort. For each trial, subjects were thoroughly instructed to act as forcefully and as fast as possible. The force signal perpendicular to the force plate and the EMG signals were synchronously sampled at $500 \mathrm{~Hz}$. The raw force signals were analog-to-digital converted and stored on a PC. During later offline analysis, the MIF trial was selected and the force signal was filtered by a digital fourth-order recursive Butterworth lowpass filter, at a cut-off frequency of $50 \mathrm{~Hz}$. Onset of force was determined at $2 \%$ of each individual's MIF. MIF and rate of force development (RFD) were calculated from the individual maximal isometric force development record. MIF was defined as the maximal voluntary force value of the force-time curve, determined in isometric conditions. $\mathrm{RFD}_{\max }$ was defined as the maximal slope at deflection of the force-time curve $\left(\Delta_{\text {force }} / \Delta_{\text {time }}\right)$. $\mathrm{RFD}_{30}$ and $\mathrm{RFD}_{100}$ were also calculated as the mean slope of the force-time curve over the time-intervals 0-30 $\mathrm{ms}$ and $0-100 \mathrm{~ms}$ (17) (Fig. 2). This protocol has recently been described in detail elsewhere (18).

\section{Electromyography (EMG)}

Circular bipolar surface electrodes (Hellige ${ }^{\circledR}$, type $44008347 \mathrm{Ag} / \mathrm{AgCl}$, diameter $10 \mathrm{~mm}$, center-to-center

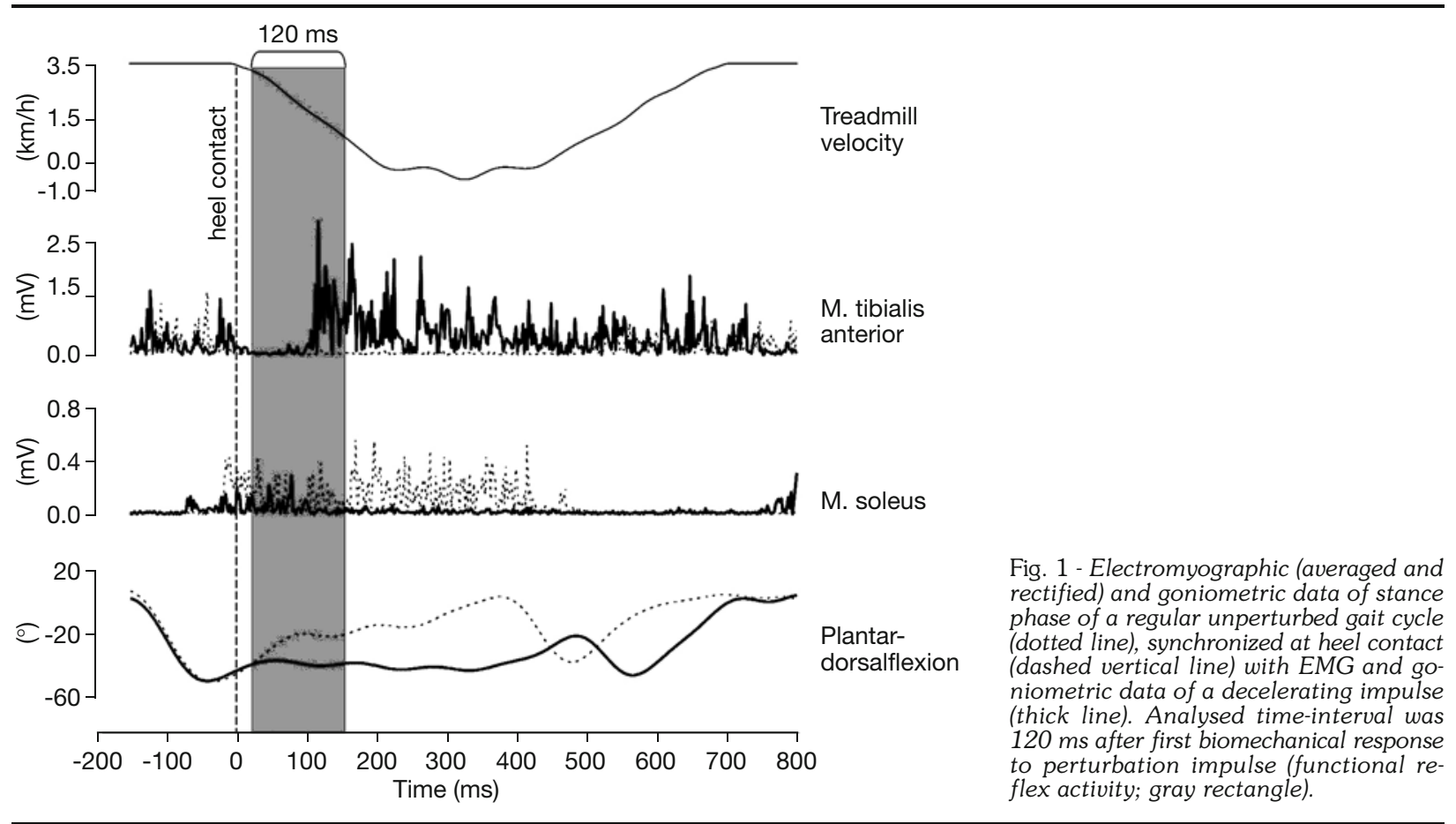




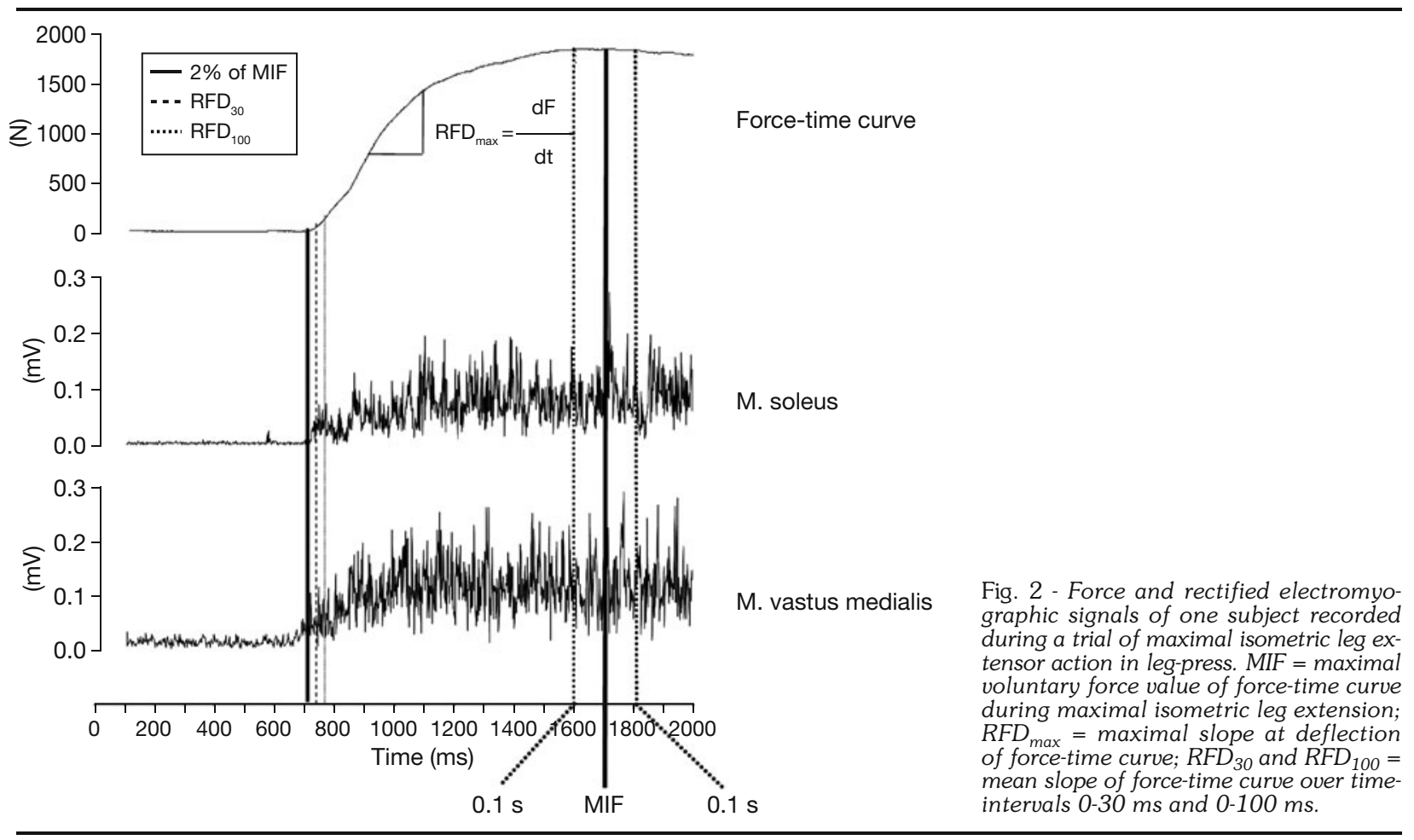

distance $25 \mathrm{~mm}$, Freiburg, Germany) were placed over the $\mathrm{m}$. tibialis anterior (TA), $\mathrm{m}$. soleus (SO) and $\mathrm{m}$. vastus medialis (VM) of the right leg. Electrodes were positioned on the muscle according to the European recommendations for surface electromyography (19). The longitudinal axes of the electrodes were in line with the direction of the underlying muscle fibers. For this purpose, the two anatomical landmarks described for the respective muscles were determined and a line was drawn between them. Electrodes were located along this line. The reference electrode was attached to the patella. Inter-electrode resistance was kept below $5 \mathrm{k} \Omega$ by shaving, slightly roughening, degreasing and disinfecting the skin. Pulling artifacts were avoided by properly fixing the electrode cables with tape to the skin. EMG signals were sampled at $500 \mathrm{~Hz}$, amplified and highpass-filtered at $10 \mathrm{~Hz}$, and carefully monitored for artifacts and noise (e.g., visual inspection of the zero line). EMG data were quantified by integrating and time-normalizing the full-wave rectified EMG signals (mean amplitude voltage [MAV]). In order to compare EMG data between the two experimental groups, the MAV of TA, SO and VM during the decelerating impulse (functional reflex activity) were normalized on the swing phase (TA) and stance phase (SO, VM) of ten averaged regular gait cycles. Thus, FRA during ten decelerating impulses was expressed in per cent of muscle activity during ten regular gait cycles. Antagonist coactivation was ex- pressed for gait perturbations as the ratio between normalized MAV of SO and normalized MAV of TA, measured during the same period of time (20). This protocol has recently been described in detail elsewhere (15). Regarding the maximal isometric force development record, MAV of SO and VM were analysed in the time-intervals 0-30 ms and 0-100 ms and normalized on MAV of SO and VM 100 ms pre- and post-MIF (Fig. 2).

\section{Statistical analyses}

Data are presented as group mean values \pm standard deviations (SD). Differences between the two groups were assessed by means of multivariate analysis of variance (MANOVA) after testing of normal distribution (Kolmogorov-Smirnov tests). Pearson's product-moment correlation coefficient was used to verify any relationship between parameters of leg extensor strength and functional reflex activity during gait perturbations in young and elderly men. Associations are reported by their correlation coefficient and level of significance. Significance level was set at $p<0.05$. All analyses were performed with the Statistical Package for Social Sciences (SPSS) version 16.0.

\section{RESULTS}

No statistically significant differences in anthropometric data (body mass, body height, BMI) were observed between young and elderly men. 

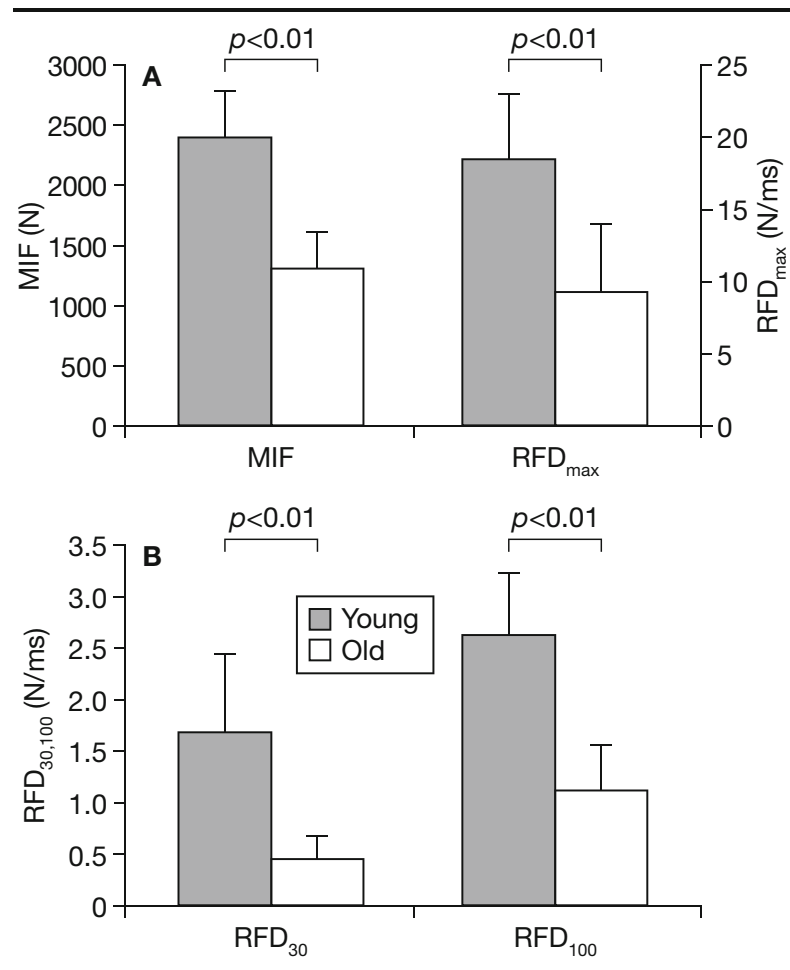

Fig. 3 - A) Maximal isometric leg extension force (MIF), expressed in $N$ and maximal rate of force development $\left(R F D_{\text {max }}\right)$, expressed in $N / m s$, presented as means $+S D$, in young and elderly subjects. Group differences are indicated by p-values.

B) Mean slope of force-time curve over time-intervals $0-30 \mathrm{~ms}$ $\left(R F D_{30}\right)$ and $0-100 \mathrm{~ms}\left(R F D_{100}\right)$, expressed in $\mathrm{N} / \mathrm{ms}$ and presented as means $+S D$, in young and elderly subjects. Group differences are indicated by $\mathrm{p}$-values.

\section{Force production}

MIF, RFD $\mathrm{Rax}_{\text {max }}, \mathrm{RFD}_{30}$ and $\mathrm{RFD}_{100}$ were significantly reduced in old compared with young subjects (MIF: $1307 \pm 312$ vs $2390 \pm 386 \mathrm{~N}, \Delta 45 \%, p<0.01 ; \mathrm{RFD}_{\max }$ : $9 \pm 5$ vs $18 \pm 4 \mathrm{~N} / \mathrm{ms}, \Delta 50 \%, p<0.01 ; \mathrm{RFD}_{30}: 0.4 \pm 0.2$ us $1.7 \pm 0.8 \mathrm{~N} / \mathrm{ms}, \Delta 76 \%, p<0.01 ; \mathrm{RFD}_{100}: 1.1 \pm 0.5$ us $2.7 \pm 0.6 \mathrm{~N} / \mathrm{ms}, \Delta 59, p<0.01$ ) (see Fig. $3 \mathrm{~A}$ and $3 \mathrm{~B}$ ). During the time-intervals $0-30 \mathrm{~ms}$ and $0-100 \mathrm{~ms}$, significant differences in normalized MAV of SO and VM were assessed between old and young subjects $\left(\mathrm{RFD}_{30}(\mathrm{SO})\right.$ : $71 \pm 90$ us $395 \pm 269 \%, \Delta 82 \%, p<0.01 ; R^{2} D_{30}$ (VM): $77 \pm 51$ vs $478 \pm 283 \%, \Delta 84 \%, p<0.01$; $\mathrm{RFD}_{100}(\mathrm{SO})$ : $81 \pm 59$ vs $449 \pm 270 \%, \Delta 82 \%, p<0.01 ; \mathrm{RFD}_{100}$ (VM): $86 \pm 50$ us $492 \pm 111 \%, \Delta 82 \%, p<0.01$ ) (Fig. 4).

Functional reflex activity during gait perturbations

Regarding decelerating perturbation impulses on the treadmill, no statistically significant differences were found for latencies in the prime mover (TA) in old subjects compared with young (78 \pm 13 vs $75 \pm 9 \mathrm{~ms}, p=0.54)$. Mean normalized MAV of TA during the decelerating per- turbation impulse (functional reflex activity) was significantly reduced for TA but not for SO or VM in old subjects compared with young (TA: $216 \pm 66$ vs $306 \pm 145 \%$, $\Delta 29 \%, p<0.05$; SO: $64 \pm 12$ vs $68 \pm 20 \%, p=0.52$; VM: $167 \pm 49$ us $189 \pm 134 \%, p=0.57$ ) (see Fig. 5). A tendency towards significance was found for levels of antagonist coactivation during gait perturbation, in terms of higher coactivation in old compared with young subjects ( $31 \pm 8$ us $25 \pm 9 \%, p=0.07$ ) (Fig. 5). No statistically significant differences in maximal angular velocity of the ankle joint during gait perturbations were detected for the old subjects compared with young $\left(96 \pm 35\right.$ vs $113 \pm 52^{\circ} / \mathrm{s}$, $p=0.30$ ).

Relationship between measures of force production and functional reflex activity during gait perturbations

No statistically significant relationship was observed between various parameters of force production and FRA in young or elderly men (Table 1).

\section{DISCUSSION}

This study investigated maximal and explosive force production capacity, as well as the ability to compensate for gait perturbations, in young and elderly men. The main findings were that: 1) maximal and explosive force production were reduced in elderly compared with young men; 2) neural activation of the respective muscles was impaired in elderly compared with young men; 3) reflex activity during gait perturbations was reduced in elderly compared with young men; 4) no statistically significant relationship was detected between measures of maximal and explosive force production and functional reflex activity during gait perturbations.

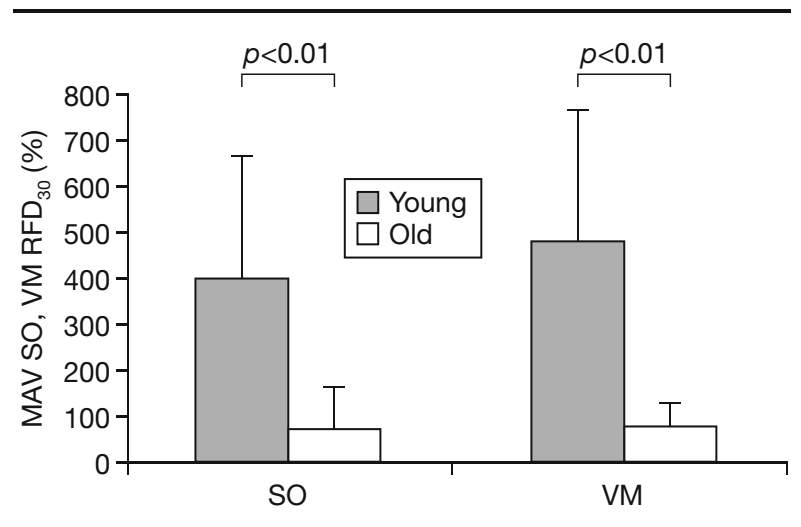

Fig. 4 - Mean amplitude voltage (MAV) of $m$. soleus (SO) and $m$. vastus medialis (VM) during maximal isometric leg extension, analysed in the time-interval 0-30 ms and normalized on mean amplitude voltage of $\mathrm{m}$. soleus and $\mathrm{m}$. vastus medialis $100 \mathrm{~ms}$ preand post-maximal isometric leg extension force, in young and elderly subjects. Data are expressed in \% and presented as means $+S D$. Group differences are indicated by p-values. 


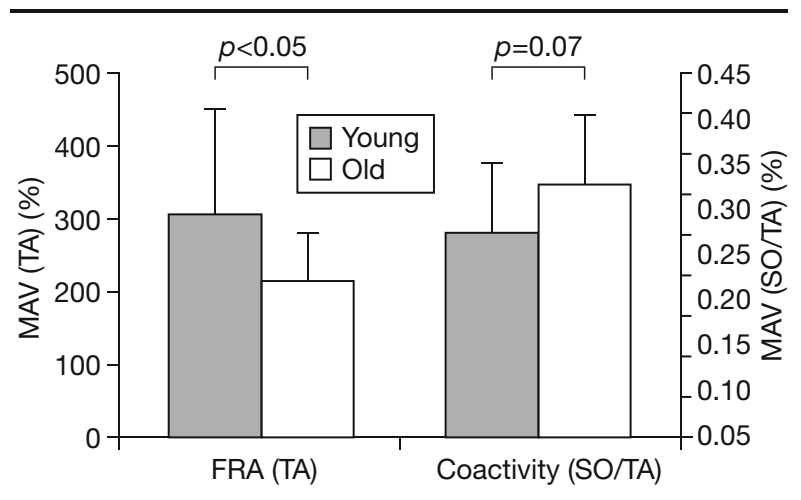

Fig. 5 - Neural activation of $m$. tibialis anterior (functional reflex activity (FRA)) and coactivity of $m$. soleus and $m$. tibialis anterior during compensation of a decelerating perturbation impulse, analysed in time-interval 0-120 ms in young and elderly subjects. Mean amplitude voltage (MAV) of $m$. tibialis anterior (TA) during compensation of a decelerating perturbation impulse, analysed in time-interval 0-120 ms and normalized on muscle activity of TA during swing phase of a regular gait cycle. Ratio in normalized mean amplitude voltage (MAV) of $m$. soleus (SO) and $m$. tibialis anterior (TA) during compensation of a decelerating perturbation impulse was calculated. MAV of TA was normalized on muscle activity of TA during swing phase of a regular gait cycle, MAV of SO was normalized on muscle activity of SO during stance phase of a regular gait cycle. Data are expressed in \% and presented as means $+S D$. Group differences are indicated by p-values.

\section{Force production}

A detailed examination of the early part of the force time curve, recorded during maximal isometric contrac- tion of the leg extensors, indicated that the very beginning of force production is especially impaired in elderly compared with younger men. The observed age-related decrease in explosive force production capacity complies with data in the literature (6), and seems to be caused by deficits in neural activation of the agonist muscles. This matches literature results, as Macaluso et al. (21) observed significant decreases in the neuromuscular activity of the $\mathrm{m}$. vastus lateralis and $\mathrm{m}$. biceps femoris during maximum isometric contraction of the knee extensors and flexors in elderly (mean 69 yrs) vs young (mean 23 yrs) healthy active women. The lower level of muscle strength in older men in this study cannot only be explained by neural deficits. Smaller contractile muscle mass and changes in muscle architecture may also contribute to the observed decreases in strength performance, although we did not explicitly investigate muscle cross-sectional area and architecture (22).

\section{Functional reflex activity during gait perturbations}

Elderly men showed smaller magnitudes of functional reflex activity in TA (prime mover) than young men, when postural control was threatened by gait perturbations.

The application of decelerating perturbation impulses at heel strike while walking on a treadmill induces an everyday stumbling stimulus, which moves the body's center of mass closer to the edge of the base of support. As a reactive response, prime mover TA is activated, to bring the body's center of mass back over the base of support. In this study, no statistically significant differences in onset latencies

Table 1 - Correlations between various parameters of force production capacity and functional reflex activity in young and elderly men.

\begin{tabular}{|c|c|c|c|c|c|c|c|c|}
\hline & MIF & $\mathbf{R F D}_{\max }$ & $\mathbf{R F D}_{30}$ & RFD $_{100}$ & $\begin{array}{c}\text { SO } \\
\text { RFD }_{30}\end{array}$ & $\begin{array}{c}\text { VM } \\
\text { RFD }_{30}\end{array}$ & $\begin{array}{c}\text { SO } \\
\text { RFD }_{100}\end{array}$ & $\begin{array}{c}\text { VM } \\
\text { RFD }_{100}\end{array}$ \\
\hline \multicolumn{9}{|l|}{ Young Men } \\
\hline Latency & 0.114 & -0.145 & 0.066 & -0.191 & -0.437 & 0.419 & -0.032 & 0.027 \\
\hline FRA in TA & -0.039 & -0.054 & 0.021 & -0.303 & -0.418 & -0.098 & -0.354 & -0.004 \\
\hline FRA in SO & -0.213 & 0.186 & 0.228 & -0.051 & -0.396 & 0.298 & -0.223 & -0.283 \\
\hline FRA in VM & 0.094 & 0.033 & -0.226 & 0.062 & 0.335 & -0.088 & 0.086 & 0.255 \\
\hline Coactivity (SO/TA) & -0.168 & 0.017 & -0.013 & 0.110 & 0.145 & 0.234 & 0.244 & -0.216 \\
\hline Maximal angular velocity & -0.288 & -0.205 & 0.119 & -0.361 & -0.299 & 0.399 & -0.167 & 0.142 \\
\hline \multicolumn{9}{|l|}{ Elderly Men } \\
\hline Latency & -0.390 & -0.177 & -0.190 & -0.243 & 0.395 & 0.379 & 0.305 & -0.081 \\
\hline FRA in TA & -0.080 & 0.359 & -0.095 & 0.256 & -0.168 & -0.060 & -0.148 & 0.314 \\
\hline FRA in $\mathrm{SO}$ & -0.194 & 0.254 & -0.425 & -0.076 & -0.314 & -0.020 & -0.282 & 0.303 \\
\hline FRA in VM & 0.288 & -0.068 & -0.016 & -0.059 & 0.261 & 0.072 & 0.072 & -0.025 \\
\hline Coactivity (SO/TA) & -0.078 & -0.229 & -0.265 & -0.385 & -0.093 & 0.016 & -0.086 & -0.334 \\
\hline Maximal angular velocity & 0.062 & 0.166 & -0.305 & 0.204 & 0.306 & 0.131 & 0.532 & 0.251 \\
\hline
\end{tabular}

FRA in TA = functional reflex activity during gait perturbations in $\mathrm{m}$. tibialis anterior; FRA in $\mathrm{SO}=$ functional reflex activity during gait perturbations in $\mathrm{m}$. soleus; FRA in VM=functional reflex activity during gait perturbations in $\mathrm{m}$. vastus medialis; $\mathrm{MIF}=$ maximal isometric leg extension force; $\mathrm{RFD}_{\max }=$ maximal rate of force development; $\mathrm{RFD}_{30}=$ mean slope of force-time curve over time-interval 0-30 ms; $\mathrm{RFD}_{100}=$ mean slope of force-time curve over time-interval 0-100 $\mathrm{ms} ; \mathrm{SO} \mathrm{RFD}_{30}=$ normalized mean amplitude voltage of $\mathrm{m}$. soleus during maximal isometric leg extension in time-interval $0-30 \mathrm{~ms} ; \mathrm{VM} \mathrm{RFD}_{30}=$ normalized mean amplitude voltage of $\mathrm{m}$. vastus medialis maximal isometric leg extension in time-interval $0-30 \mathrm{~ms} ; \mathrm{SO}_{\mathrm{RFD}} \mathrm{H}_{100}=$ normalized mean amplitude voltage of $\mathrm{m}$. soleus during maximal isometric leg extension in time-interval $0-100 \mathrm{~ms} ; \mathrm{VM} \mathrm{RFD}_{100}=$ normalized mean amplitude voltage of $\mathrm{m}$. vastus medialis maximal isometric leg extension in time-interval 0-100 ms. 
in TA following perturbation impulses were detected between young or old subjects. This is in accordance with a study by Woollacott et al. (23), who observed that healthy older subjects did not show significant slowing in onset latency in response to threats to balance compared with young subjects. However, Lin and Woollacott (24) compared unstable and stable elder adults with young adults, and found that significant slowing in onset latency following postural threats was only observed in unstable elder adults. The reported high physical activity levels of the elderly men in the present study imply that our subjects can be classified as stable. This may explain why we did not find significant slowing in onset latencies.

Slower onset latencies, alone, do not threaten the equilibrium of the body's center of mass. However, this seems to be the case when slower onset latencies and inefficient postural responses coincide, which was partly investigated in this study. The impaired ability of our elderly men to compensate for gait perturbations matches literature results. Tang and Woollacott (25) found smaller magnitudes of postural responses to unexpected slips during walking in older adults. Lin and Woollacott (24) investigated smaller integrated EMG activity in muscles compensating for backward surface translations in stable and unstable older adults compared with young adults. Older adults, whose postural response capacity may be limited by slowness (reduced RFD) and weakness (reduced MIF), probably need to modify their postural muscle responses differently from young adults in order to maintain postural stability. This compensatory mechanism can be achieved by various movement strategies (ankle, hip, and step strategies) (23) and by increasing the magnitude of antagonist coactivation (25). In the present study, old subjects showed a tendency toward higher levels of antagonist coactivation in muscles (TA, SO), compensating for the decelerating perturbation impulses. It may be argued that the high physical activity level of our subjects and/or the small sample size accounts for the fact that parameter antagonist coactivation did not reach the level of significance. Tang and Woollacott (25) found higher levels of antagonist coactivation in elder adults compared with young adults when responding to gait perturbations. Nielsen and Kagamihara (26) suggested that there is a specific motor program for coactivation and that interneurons in the reciprocal pathway are actively inhibited by a central source during coactivation. This depression of reciprocal inhibition leads to a high level excitability of the motoneurons of the antagonist muscles, and results in coactivation. The depression is most probably centrally mediated (27).

Relationship between measures of force production and functional reflex activity during gait perturbations

We found no statistically significant relationship in young or elderly men between measures of maximal and explosive force production, neural activation of the re- spective muscles, and functional reflex activity during gait perturbations. This finding is of particular importance for the elderly population, because of the underlying implications for exercise. Thus, the present results will primarily be discussed for elderly men. In addition, the relationship between measures of strength and balance has already been investigated in young adults. No statistically significant association was detected (28).

At first sight, our findings seem to be contradictory to data in the literature. Other investigators have found significant correlations between lower extremity strength in old age and the ability to recover after an unexpectedly induced trip $(12,29)$. The reason for this discrepancy is probably to be found in the differing study methods applied. We investigated the ability to compensate for decelerating gait perturbations on a treadmill by means of electromyographic and goniometric analysis. Pavol et al. (12) and Pijnappels et al. (29) induced trips over obstacles that unexpectedly appeared from the ground while subjects were walking at a self-selected speed. Kinematic data, ground reaction forces and centre of pressure of the support limb were analysed in the study by Pijnappels et al. (29). Pavol et al. (12) simply classified the outcome of each trip in four categories: 1) recovery from a trip; 2) fall after a trip; 3) support by safety harness after a trip; 4) no trip due to missed obstacle. No kinematic, electromyographic or dynamographic analyses were conducted. However, isometric and isokinetic force production of the ankle, knee, and hip joints were assessed, in order to establish an association between strength performance and the ability to recover after a postural perturbation. Therefore, the primary outcome measures of the two above studies may primarily be allocated to a performance level, whereas ours are predominantly on a neuromuscular level. In addition, it seems plausible that the impact of strength in the compensation of postural perturbation impulses varies with the type of applied perturbation and the phase of perturbation analysed. In our study, decelerating perturbation impulses were applied while subjects were walking on a treadmill at a speed of $3.5 \mathrm{~km} / \mathrm{h}$. Furthermore, the initial perturbation phase was investigated, which is predominantly regulated by reflex movements. There is in fact evidence that gait perturbations are most probably compensated for by polysynaptic reflexes via group II afferents (30). In the studies by Pavol et al. (12) and Pijnappels et al. (29), subjects were tripped while walking at a self-selected speed and the late recovery phase was observed. It is well documented that long-latency responses after balance threats are cortically mediated in terms of direct corticospinal pathways (31). This may explain why an association was found in the above studies between strength performance and the recovery phase of a perturbation impulse (partly voluntary muscle action), but not in the present study, which investigated the initial phase of a pos- 
tural perturbation (primarily involuntary or reflex muscle action).

Three potential limitations of this study warrant discussion. First, we assessed maximal and explosive force production capacity in the leg extensors while FRA was evaluated in the TA. We acknowledge that muscle behavior may be completely different in leg extensors and TA. Regarding leg extensors, it is well known that strength performance is closely related to the performance of ADLs (activities of daily living) $(32,33)$. However, there is no clear indication in the literature that the strength performance of the dorsiflexors (e.g., TA) influences the successful execution of ADLs. In this regard, only weak correlations have been found between the maximal isometric strength of dorsiflexors and gait performance, as well as postural sway, determined by computerized posturography (34). Again, it is reported that the dorsiflexors play an important role in compensating gait perturbations (25). Therefore, predominantly functional and not biomechanical reasons made us choose to determine the force production capacity of the leg extensors and FRA in TA. In addition, no significant correlations were detected between FRA in VM and various parameters of strength performance. This seems to reinforce our methodological approach, since VM plays a major role in both force production of the leg extensors and compensation of gait perturbations.

Second, we tested for isometric leg extension force, while there is evidence in the literature that there is a particularly close association between leg extensor power and functional performance (e.g., chair rise, stair climb) in elderly subjects (35). However, Bean et al. (36) found similar associations in subjects aged 65 and older between chair rise time and leg power $(\mathrm{r}=0.27)$ on one hand, and chair rise time and isometric knee $(\mathrm{r}=0.26)$ and hip strength $(r=0.27)$ on the other. In addition, leg power was associated with hip strength $(\mathrm{r}=0.76)$ and knee strength $(r=0.73)$ (36). Thus, the functional relevance of the present study seems to be given.

Third, the cross-sectional character of this study is a further limitation. A causal relationship between men's age and observed strength/reflex performance cannot be inferred. Therefore, care is needed when generalizing the present findings to other kinds of testing situations or groups of subjects.

\section{CONCLUSIONS}

Questions remain regarding the functional implication of our results. From a fall-preventive or therapeutic point of view, it is of great interest to know which training interventions can counteract the investigated detrimental effects. The results of the present study indicate that strength performance and FRA are two independent neuromuscular capacities which deteriorate in old age. Thus, it seems plausible to argue that they should be trained complementarily. It is evident from the literature that researchers are increasingly studying the effects of more specifically designed training programs on neuromuscular performance in populations of older adults. Recent studies indicate that strength training combined with modified power types of exercises have a greater impact on explosive force production capacity and ADL in old age than traditional heavy resistance strength training (37). In addition, exercise programs that include balance training components have tended to be most effective as regards their impact on postural control (13). Preliminary results indicate that balance training has an effect on functional reflex activity during gait perturbations in seniors (13). Recent studies also suggest that the most effective fall prevention training programs should incorporate specifically designed perturbation modes $(38,39)$. This new approach is based on the assumption that neural control of volitional limb movements differs in some fundamental ways to reactions evoked by postural perturbation (40). Thus, the results of the present study seem to support the tendency toward more specifically designed training programs.

In summary, in this study maximal and explosive force production capacity and functional reflex activity during gait perturbations were examined in a cohort of young and elderly men. Elderly men showed a greatly reduced maximal and explosive force production capacity, and the TA EMG response evoked by gait perturbations was impaired in old compared with young adults. No significant relationship was observed between strength performance and FRA during gait perturbations. Thus, it is hypothesized that these important functional capacities for seniors must be trained complementarily. Future studies should extend the results of this study to healthy active older adults by including fall-prone and sedentary subjects, as well as subjects with chronic diseases (e.g., dementia, Parkinson).

\section{ACKNOWLEDGEMENTS}

The authors would like to thank Dr. Harald Seelig for his statistical advice and Dr. Dieter Strass for his contributions to the design of the study.

Funding sources and Disclosure: Our sponsor (LGFG Grant (Landesgraduiertenförderung)), Baden-Württemberg, Germany did not have any influence on the design, methods, subject recruitment, data collections, analysis or preparation of this paper. The endorsement involved a scholarship (monthly salary) for the corresponding author from a state program.

\section{REFERENCES}

1. Izquierdo M, Aguado X, Gonzalez R, Lopez JL, Häkkinen K. Maximal and explosive force production capacity and balance performance in men of different ages. Eur J Appl Physiol 1999; 79: 260-7.

2. Granacher U, Zahner L, Gollhofer A. Strength, power, and postural control in seniors: considerations for functional adaptations and for fall prevention. Eur J Sports Sci 2008; 8: 325-40.

3. Metter EJ, Lynch N, Conwit R, Lindle R, Tobin J, Hurley B. Muscle quality and age: cross-sectional and longitudinal comparisons. J Gerontol A Biol Sci Med Sci 1999; 54: B207-18. 
4. Akima H, Kano Y, Enomoto Y et al. Muscle function in 164 men and women aged 20-84 yr. Med Sci Sports Exerc 2001; 33: 220-6.

5. Lynch NA, Metter EJ, Lindle RS et al. Muscle quality. I. Age-associated differences between arm and leg muscle groups. J Appl Physiol 1999; 86: 188-94.

6. McNeil CJ, Vandervoort AA, Rice CL. Peripheral impairments cause a progressive age-related loss of strength and velocity-dependent power in the dorsiflexors. J Appl Physiol 2007; 102: 1962-8.

7. Muller F, Dehail P, Bestaven E et al. Maximal and sustained isokinetic lower-limb muscle strength in hospitalized older people. Muscle Nerve 2007; 35: 739-44.

8. Klitgaard H, Mantoni M, Schiaffino S et al. Function, morphology and protein expression of ageing skeletal muscle: a cross-sectional study of elderly men with different training backgrounds. Acta Physiol Scand 1990; 140: 41-54.

9. Era P, Sainio P, Koskinen S, Haavisto P, Vaara M, Aromaa A. Postural balance in a random sample of 7,979 subjects aged 30 years and over. Gerontology 2006; 52: 204-13.

10. Pavol MJ, Runtz EF, Edwards BJ, Pai YC. Age influences the outcome of a slipping perturbation during initial but not repeated exposures. J Gerontol 2002; 57: M496-503.

11. Pijnappels M, van der Burg PJ, Reeves ND, van Dieen JH. Identification of elderly fallers by muscle strength measures. Eur J Appl Physiol 2008; 102: 585-92.

12. Pavol MJ, Owings TM, Foley KT, Grabiner MD. Influence of lower extremity strength of healthy older adults on the outcome of an induced trip. J Am Geriatr Soc 2002; 50: 256-62.

13. Granacher U, Gollhofer A, Strass D. Training induced adaptations in characteristics of postural reflexes in elderly men. Gait Posture 2006; 24: 459-66.

14. Frey I, Berg A, Grathwohl D, Keul J. Freiburg Questionnaire of physical activity - development, evaluation and application. Soz Praventivmed 1999; 44: 55-64.

15. Granacher U, Gruber M, Gollhofer A. The impact of sensorimotor training on postural control in elderly men. Deut Z Sportmed 2009; 60: 16-22.

16. Pijnappels M, Bobbert MF, van Dieen JH. EMG modulation in anticipation of a possible trip during walking in young and older adults. J Electromyogr Kinesiol 2006; 16: 137-43.

17. Gruber M, Gollhofer A. Impact of sensorimotor training on the rate of force development and neural activation. Eur J Appl Physiol 2004; 92: 98-105.

18. Granacher U, Gruber M, Gollhofer A. Resistance training and neuromuscular performance in seniors. Int J Sports Med 2009; 30: 652-7.

19. Hermens HJ, Freriks B, Merletti R et al. European recommendations for surface electromyography: results of the SENIAM project. Roessingh Research and Development bv, 1999.

20. Klass M, Baudry S, Duchateau J. Aging does not affect voluntary activation of the ankle dorsiflexors during isometric, concentric, and eccentric contractions. J Appl Physiol 2005; 99: 31-8.

21. Macaluso A, Nimmo MA, Foster JE, Cockburn M, McMillan NC, De Vito G. Contractile muscle volume and agonist-antagonist coactivation account for differences in torque between young and older women. Muscle Nerve 2002; 25: 858-63.

22. Narici MV, Maganaris CN, Reeves ND, Capodaglio P. Effect of aging on human muscle architecture. J Appl Physiol 2003; 95: 2229-34.

23. Woollacott MH, Shumway-Cook A, Nashner LM. Aging and posture control: changes in sensory organization and muscular coordination. Int J Aging Hum Dev 1986; 23: 97-114.

24. Lin SI, Woollacott MH. Postural muscle responses following changing balance threats in young, stable older, and unstable older adults. J Mot Behav 2002; 34: 37-44.

25. Tang PF, Woollacott MH. Inefficient postural responses to unexpected slips during walking in older adults. J Gerontol A Biol Sci Med Sci 1998; 53: M471-80.

26. Nielsen J, Kagamihara Y. The regulation of disynaptic reciprocal Ia inhibition during co-contraction of antagonistic muscles in man. J Physiol 1992; 456: 373-91.

27. Rothmuller $\mathrm{C}$, Cafarelli E. Effect of vibration on antagonist muscle coactivation during progressive fatigue in humans. J Physiol 1995; 485: 857-64.

28. McCurdy K, Langford G. The relationship between maximum unilateral squat strength and balance in young adult men and women. J Sports Sci Med 2006; 5: 282-8.

29. Pijnappels M, Bobbert MF, van Dieen JH. Push-off reactions in recovery after tripping discriminate young subjects, older non-fallers and older fallers. Gait \& Posture 2005; 21: 388-94.

30. Grey MJ, Ladouceur M, Andersen JB, Nielsen JB, Sinkjaer T. Group II muscle afferents probably contribute to the medium latency soleus stretch reflex during walking in humans. J Physiol 2001; 534: 925-33.

31. Taube W, Schubert M, Gruber M, Beck S, Faist M, Gollhofer A. Direct corticospinal pathways contribute to neuromuscular control of perturbed stance. J Appl Physiol 2006; 101: 420-9.

32. Bean JF, Kiely DK, Herman S et al. The relationship between leg power and physical performance in mobility-limited older people. J Am Geriatr Soc 2002; 50: 461-7.

33. Skelton DA, Greig CA, Davies JM, Young A. Strength, power and related functional ability of healthy people aged 65-89 years. Age Ageing 1994; 23: 371-7.

34. Ringsberg K, Gerdhem P, Johansson J, Obrant KJ. Is there a relationship between balance, gait performance and muscular strength in 75-year-old women? Age Ageing 1999; 28: 289-93.

35. Bassey EJ, Fiatarone MA, O'Neill EF, Kelly M, Evans WJ, Lipsitz LA. Leg extensor power and functional performance in very old men and women. Clin Sci 1992; 82: 321-7.

36. Bean JF, Leveille SG, Kiely DK, Bandinelli S, Guralnik JM, Ferrucci L. A comparison of leg power and leg strength within the InCHIANTI study: which influences mobility more? J Gerontol 2003; 58: 728-33.

37. Sayers SP. High-speed power training: a novel approach to resistance training in older men and women. A brief review and pilot study. J Strength Cond Res 2007; 21: 518-26.

38. Maki BE, Cheng KC, Mansfield A et al. Preventing falls in older adults: new interventions to promote more effective change-in-support balance reactions. J Electromyogr Kinesiol 2008; 18: 243-54.

39. Sakai M, Shiba Y, Sato H, Takahira N. Motor adaptations during slip-perturbed gait in older adults. J Phys Ther Sci 2008; 20: 109-15.

40. Maki BE, Mcllroy WE. Change-in-support balance reactions in older persons: an emerging research area of clinical importance. Neurol Clin 2005; 23: 751-83. 